\title{
A new synthesis algorithm based on least squares for InSAR phase unwrapping
}

\author{
Liubing Jiang ${ }^{1,2, \text { a }}$, Yongji Liü, ${ }^{3, b}$, Li Che ${ }^{1,2, c}$,Cong Li $i^{4, d}$ \\ ${ }^{1}$ School of Computer Science and Information Security, Guilin University of Electronic \\ Technology, Guilin, Guangxi 541004, China \\ ${ }^{2}$ Guangxi Wireless Broadband Communication and Signal Processing Key Laboratory, Guilin \\ University of Electronic Technology, Guilin 541004, China \\ ${ }^{3}$ School of Information and Communication, Guilin University of Electronic Technology, Guilin, \\ Guangxi 541004, China \\ ajlbnj@163.com, ${ }^{b}$ aji241@hotmail.com, ${ }^{c} j$ lrq1@163.com, ${ }^{d}$ licongxudahua815@163.com
}

\begin{abstract}
Keywords: Phase unwrapping; Least square method; Local frequency estimation; Interferometry Abstract: Phase unwrapping is a key step of interference synthetic aperture radar (InSAR) signal processing, while the existence of some factors such as the noises and the lack of data sampling makes this process extremely difficult. The least square method is the fastest way to unwrap, and there is no region that cannot be unwrapped. The unwrapping process using the least square method is to go across the residues, which leads to the propagation of error in the entire range, causing unideal unwrapping results due to the excessive errors. Here is a new algorithm of phase unwrapping aimed at the features of the least square method. The new algorithm is based on the least square method. First of all, it recognizes the residues, determining the unwrapping process tentatively by using the least square method to unwrap so as to get the preliminary results of the residues. Then, it can make effective estimation of the unwrapping value of the residues using the local frequency estimation, and continue the unwrapping process of the least square method by utilizing the values after the reconfiguration. The new algorithm adds local frequency estimation into the unwrapping process of the least square method, making effective estimation reconfiguration of the residues specifically in order to improve the unwrapping accuracy by lowering the time consumption as much as possible. Both the simulation and measured data experiments prove that the new algorithm is able to reduce the overall impact of the noise pixels, restrain the spread of the unwrapping errors and enhance the phase unwrapping accuracy.
\end{abstract}

\section{Introduction}

During the data dealing process of the Interference Synthetic Aperture Radar, phase unwrapping is a vital step in interfering the measurement [1-3]. Its precision will directly influence the accuracy of the digital elevation model produced by the InSAR [3]. There are mainly four types of ways to unwrap the interferogram: the first type is the phase unwrapping based on the path tracking[4,5]; the second type is the minimum norm[6,7]; the third type is the network-flow[8]; the fourth type is the other methods except the above three types.

The most common way in the path tracking method is the branch-cuts, which was first brought up by Goldstein[4]. He wrapped the phase data to get the location distribution map of the plus-minus residues, and then jointed the residues that were near to each other to form the branch-cut lines, so that the charges on the branch-cut lines could reach a balance, and the integral path would integrate around the plus-minus residues that could cancel each other out. After jointing the branch-cut lines, we need to unwrap all the pixels in the images one by one, revealing the true phase information. It's hard for the branch-cuts to choose accurate branch-cut lines in the regions with poor relativity and many residues. There will easily be isolated intervals, that is the unwrapping island, surrounded by the branch-cut lines, and they can't be unwrapped as the integral path even can't reach there[9]. Besides, there is no obvious connection between them, so unwrapping individually will cause great errors.

The network-flow is a better solution to get the minimization problem by searching for the best path and the shortest branch-cuts throughout the range. Under the condition of mature network-flow, the 
network planning algorithm also can improve the running efficiency[10]. In the meantime, it can ensure certain precision, restraining errors from being spread to the entire region. And as for the relatively severe interferogram, it also can carry out unwrapping and receive quite accurate results[11-13]. However, if the environment is too noisy, the unwrapping results of the network-flow will inevitably propagate the noise along the interval path, leading to serious peak phenomenon in the unwrapping results. What's more, a large amount of internal storage will be consumed when in face of the relatively large interferogram, and it's difficult for the restrain of the unwrapping speed to be applied to the practical engineering in reality[12-14].

The least square method is the most classic minimum norm. It belongs to an overall algorithm. There is no region that can't be unwrapped in the phase unwrapping results, but because this algorithm will cross instead of passing by the residues, it can spread the phase errors to the entire regions easily, which therefore results into the low unwrapping accuracy because of the noise propagation throughout the range [9].

Focusing on the speedy algorithm of the least square method, this thesis adds local frequency estimation algorithm and the least surface fitting algorithm into the unwrapping process so as to lower and restrain the noise errors caused by residues. It can improve the unwrapping accuracy with low time consumption and make sure of the highly effective algorithm at the same time. This passage will first introduce some relative theories and then talk about the detailed process and derivation of the new algorithm, and at last, verify its feasibility through experiments.

\section{The improved algorithm}

Least square method for unwrapping: The basic idea of the least square method is to solve the minimum deviation between the real phase gradient and the wrapping phase gradient. Assuming that $\varphi_{i, j}$ represents the wrapped phase and the phase difference between the wrapped phase on the row and the column are respectively:

$$
\begin{aligned}
\nabla_{i, j}^{x} & =\operatorname{wrap}\left\{\varphi_{i, j+1}-\varphi_{i, j}\right\} \\
\nabla_{i, j}^{y} & =\operatorname{wrap}\left\{\varphi_{i+1, j}-\varphi_{i, j}\right\}
\end{aligned}
$$

In formula (1) and (2), $i=0,1,2, \mathrm{~L} M-1 ; j=0,1,2, \mathrm{~L} N-1$ and $\operatorname{wrap}\{\bullet\}$ represents the convolution operation of the model, that is, the wrapping factor.

Assuming that $\phi(i, j)$ represents the unwrapping phase, then the least square phase unwrapping method is to minimize the formula (3):

$$
\sum_{i=0}^{M-1} \sum_{j=0}^{N}\left(\phi_{i+1, j}-\phi_{i, j}-\Delta_{i, j}^{x}\right)^{2}+\sum_{i=0}^{M} \sum_{j=0}^{N-1}\left(\phi_{i, j-1}-\phi_{i, j}-\Delta_{i, j}^{y}\right)^{2}
$$

In order to get the minimum value of formula (3), we need to find partial derivatives of $\phi_{i, j}$ in formula (1), (2) and (3) and make them zero, and we can get the following formula through derivation:

$$
\left(\phi_{i+1, j}-2 \phi_{i, j}+\phi_{i-1, j}\right)+\left(\phi_{i, j+1}-2 \phi_{i, j}+\phi_{i, j-1}\right)=\Delta_{i, j}^{x}-\Delta_{i-1, j}^{x}+\Delta_{i, j}^{y}+\Delta_{i, j-1}^{y}
$$

Assume $\Delta_{i, j}^{x}-\Delta_{i-1, j}^{x}+\Delta_{i, j}^{y}+\Delta_{i, j-1}^{y}=\rho_{i, j}$, then the formula (4) is a discrete Poisson equation meet Nenmann boundary conditions on $\mathrm{M} \times \mathrm{N}$ grid:

$$
\frac{\partial^{2}}{\partial x^{2}} \phi_{x, y}+\frac{\partial^{2}}{\partial y^{2}} \phi_{x, y}=\rho_{x, y}
$$


By solving the formula 5, we can get the unwrapped phase, however, the least squares method does not deal with the residual points, so the unwrapping error will spread to the high quality area with the process of unwrapping, which will cause the whole image's unwrapping accuracy to decrease.

Therefore, the new algorithm adds the step of processing residual points in the least square method of unwrapping, so as to improve the precision of unwrapping.

Residual points processing: As shown in figure 1, in a schematic of the four adjacent wrapping phase. Define $\Delta_{r}$ as the entanglement phase difference on the four sides, and the result of the winding operation again is the result of the winding operation symbol, which can be obtained:

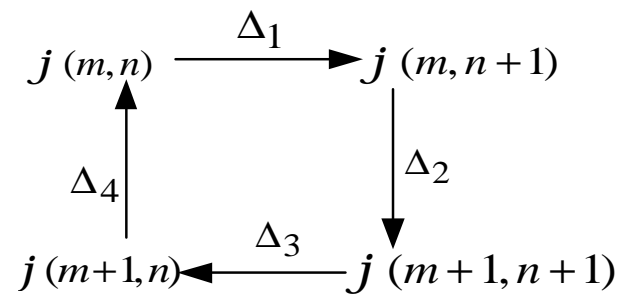

Fig.1. Four adjacent wrapping phase schematic

$$
\begin{aligned}
& \Delta_{1}=\omega[\varphi(m, n+1)-\varphi(m, n)] \\
& \Delta_{2}=\omega[\varphi(m+1, n+1)-\varphi(m, n+1)] \\
& \Delta_{3}=\omega[\varphi(m+1, n)-\varphi(m+1, n+1)] \\
& \Delta_{4}=\omega[\varphi(m, n)-\varphi(m+1, n)]
\end{aligned}
$$

in the formula (6):

$$
\omega[x]=x+2 k \pi \quad-\pi<\omega[x]<\pi, \quad k \in Z
$$

Because all of $\Delta_{r}$ meet $(-\pi, \pi]$, the sum of integration must be satisfied the formula (8) as follow:

$$
I=\sum_{r=0}^{4} \Delta_{r} / 2 \pi=\left\{\begin{array}{c}
1 \\
0 \\
-1
\end{array}\right.
$$

The accuracy of the two dimensional phase unwrapping result is closely related to the selected integral path, if the integral path passes through the phase discontinuities, the unwrapping error will be get larger. Goldstein named these discontinuous phase points as residual points [4]. If $I=0$, the point $(m, n)$ is a continuous point, and the integral path of the four points is consistent, Otherwise, the $(m, n)$ point is considered to be a residual point. Under the condition of $I=-1,(m, n)$ is a negative residual point, and $(m, n)$ is a positive residual point when $I=1$.In the actual phase diagram, if the phase jump is caused by the noise of an independent point, the positive and negative residual points will appear in pairs. By adjusting the value of discontinuous edges corresponding to two points, the discontinuous points can be changed into continuous points at the same time. Therefore, the discontinuities can be eliminated by reasonably estimating the values of two discontinuous points to eliminate the effect of noise. Maximum likelihood power spectrum estimation is a high-resolution nonlinear spectral estimation method, especially for frequency, wave and power spectrum estimation of underwater acoustic and seismic waves. It can also be used for the estimation of power spectrum of stationary time series. The maximum likelihood estimation of power spectrum is slightly lower than the maximum entropy method of power spectrum estimation, but its performance is more stable fit for the value estimation of discontinuities. There are many ways to estimate the interferometric phase local frequency. The maximum likelihood frequency estimation method is one of the most widely applied methods which can be implemented by fast Fourier transform (FFT). In a small window of the complex interferogram, the slope of the terrain can be considered as a constant, that is, the complex 
interferogram contains only one main frequency, which can be represented by a two-dimensional complex sine signal. In a rectangular window, the interference phase can be written as:

$$
\phi(m+k, n+l)=2 \pi k \square f_{a}+2 \pi l f_{r}+\phi(m, n)
$$

In the formula, $\phi(m, n)$ is the phase of the center pixel of the window, $k$ and $l$ are the offset of the pixels in the window along the azimuth and distance from the center pixel, and $f_{a}$ and $f_{r}$ are the interferometric phase frequency of azimuth and distance respectively. The maximum likelihood frequency estimation method is carried out by the following formula:

$$
\left(f_{a}, f_{r}\right)=\arg \max _{\left(f_{a}, f_{r}\right)}\left\{\mid \sum_{k=-\frac{N_{a}-1}{2}}^{\frac{N_{a}-1}{2}} \sum_{t=\frac{N_{r}-1}{2}}^{\frac{N_{r}-1}{2}} \exp \left(j \phi(m+k, n+l) \exp \left(-j 2 \pi\left(f_{a} k+f_{r} l\right)\right) \mid\right\}\right.
$$

In formula (10), $f_{a}, f_{r}$ are the estimated value of $f_{a}$ and $f_{r}$, the window size is $N_{\mathrm{a}} \times N_{\mathrm{r}}$. In order to ensure the stationarity of the signal in the window, the size of the window should not be too large. Otherwise, in the process of implementation, the variance of the estimation result will be larger due to the quantization effect of two-dimensional FFT. In order to improve the estimation accuracy, without increasing the size of the window, we can add two zeros to the signal in the window, and then carry out two dimensional FFT, so as to reduce the purpose of the frequency sampling interval. According to the two-dimensional local frequency estimation variance of the Cramer-Rao bound (CRB), can use the formula (11) to calculate the zero after the window size.

$$
\left\{\begin{array}{l}
\left(\frac{2 \pi}{L_{a}}\right) \leq \frac{6}{r_{s / n} N_{a} N_{r}\left(N_{a}^{2}-1\right)} \\
\left(\frac{2 \pi}{L r}\right) \leq \frac{6}{r_{s / n} N_{a} N_{r}\left(N_{r}^{2}-1\right)}
\end{array}\right.
$$

In the formula(11), $L_{a}, L_{\mathrm{r}}$ represent zero after the range and azimuth direction of the window length , $r_{s / n}$ is the interferogram signal-to-noise ratio, inequality left is the discrete Fourier transform of output frequency resolution of the square, right said local frequency estimation variance of the CRB. Without increasing the size of the window and ensuring the speed of unwrapping, in order to improve the estimation accuracy, the following strategies are selected for selecting the window size of $4 * 4$ for paired residuals. As shown in the first rectangle of Figure 2, the $4 * 4$ pixels, which are directly surrounded by a pair of residual points, are selected as the window for the pair of pairs of residual points that appear in the same column. And for the pair of pairs that appear on the same line, select the $4 * 4$ window data as shown in the second rectangle frame of Figure 2. For the residual point of diagonal appearance, in order to reduce the time consumption, the window fetching method is still $4 *$ 4 , and third and fourth rectangular boxes are selected.

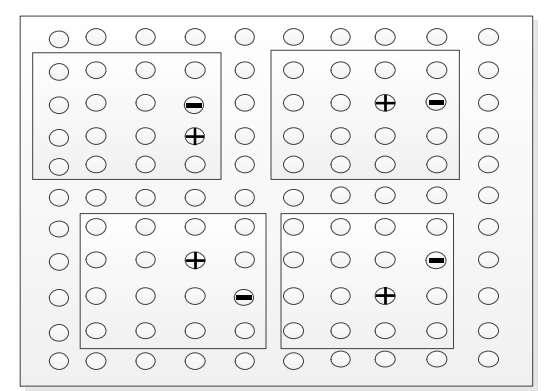

Fig.2. Window selection strategy for pairwise residual points 
New algorithm steps: In the process of least square method, it does not mark the residual points making the noise spread in the global range, and there are always some discontinuous regions. Using the local frequency estimation, the effective numerical reconstruction of the residual points is processed, and the least square method is used for unwrapping. Therefore, the local frequency estimation is integrated into the least square method, which can effectively reduce the influence of noise and improve the precision of unwrapping. Because we cannot guarantee the continuity of reconstructed values of residuals with local frequency estimation, we need to rewrap the preliminary unwrapping results, then recognize the residual points again, and do the second unwrapping operation. The detailed steps of the algorithm are as follows:

Step 1: Identify and record the position of residual points in the interferogram.

Step 2: Mark residual points, use least square method to solve the unwrapping, and get the last column of pixel regions containing residual points, and stop the unwrapping and save intermediate data.

Step 3: Use the effective value of the unwrapping area around the residual points to estimate the residual points and estimate the residual points according to the local frequency estimation.

Step 4: Rewrap the estimated value obtained by step three and returning the unwrapping value saved by step two to return to the position of the residual point and unwrapping again.

Step 5: Repeat step two to step four until the end of unwrapping is finished, and the initial unwrapping result set 1 can be obtained

Step 6: Rewrap the unwrapping results obtained from step 5.

Step 7: Repeat step 1 to step 6, and get the final unwrapping result.

\section{Experiment results}

The proposed method will be tested with synthetic data and real data to effectively evaluate the performance, and also will be compared the most used methods including the BC [14] algorithm, the NF method [8] and the LS method [9]. The run-time of the above algorithms operating in the same MATLAB environments (R2014a + 64bit) on a PC equipped with an Intel(R) Xeon(R) E3-1214 + 64 bit $\times$ Windows 7 SP1 Ultimate edition +8 GB of RAM where the run-time denotes the average values of running time computed from 50 simulation runs.

Unwrapped results of the simulated data: The simulated data shown in Fig. 3 is a $256 * 256$ phase surface generated by the peaks function provided by MATLAB. Select running time and root mean square error (RMSE) to test the algorithm, and the formula for the RMSE is formula 12.

$$
R M S E=\sqrt{\frac{\sum_{i=0}^{M-1} \sum_{j=0}^{N-1}\left(\phi_{i, j}-\varphi_{i, j}\right)^{2}}{M \times N}}
$$

The random noise and the salt-and-pepper noise with typical signal-to-noise ratio (SNR) of 6.11dB and $-0.51 \mathrm{~dB}[15]$ is added to the original data separately. Fig. 4(a) shows the wrapped phase surface with SNR of 6.11dB and Fig. 4(b) is the top view of the wrapped phase surface. The unwrapped results are shown in Fig. 5, and the experiment results are summarized in Table 1.

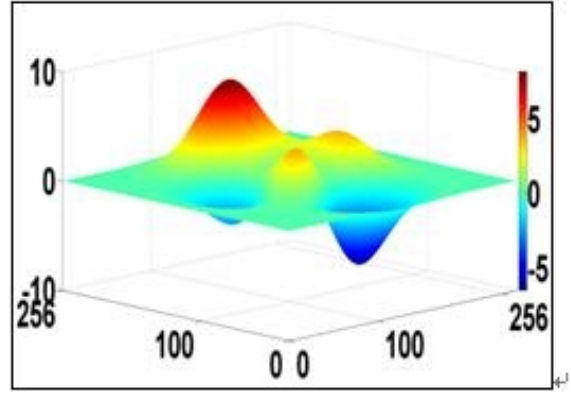

(a)

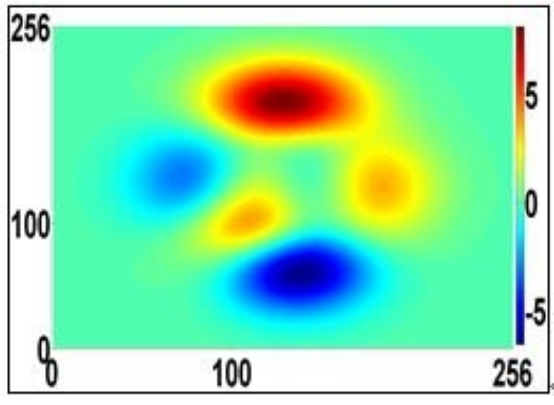

(b)

Fig.3 (a) phase surface data $\quad$ (b) top view of (a) 


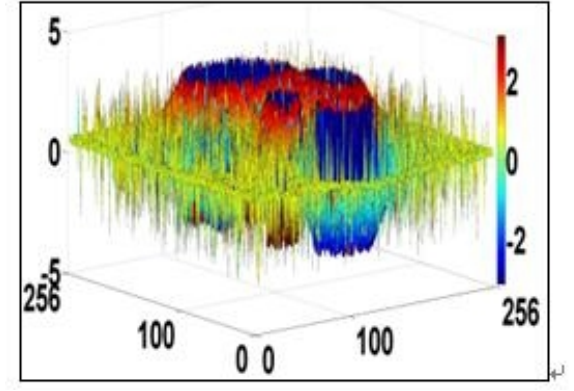

(a)

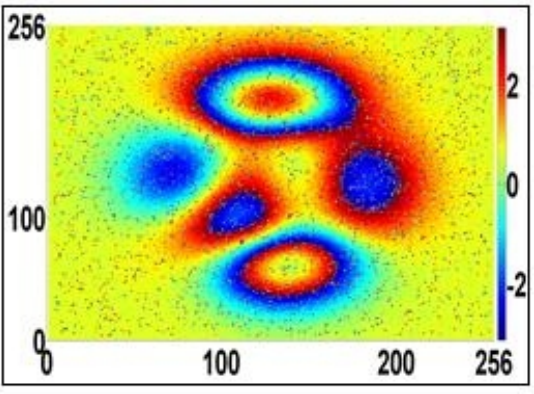

(b)

Fig. 4(a) wrapped phase with SNR of $6.11 \mathrm{~dB} \quad$ (b) top view of (a)

Table 1 Simulation results with SNR of $6.11 \mathrm{~dB}$

\begin{tabular}{|l|c|c|}
\hline Algorithm & Running Time(s) & RMSE \\
\hline branch cut & 6.3648 & 0.8079 \\
\hline least square & 0.2340 & 1.0312 \\
\hline network flow & 5.4132 & 0.6152 \\
\hline new algorithm & 1.8720 & 0.7532 \\
\hline
\end{tabular}

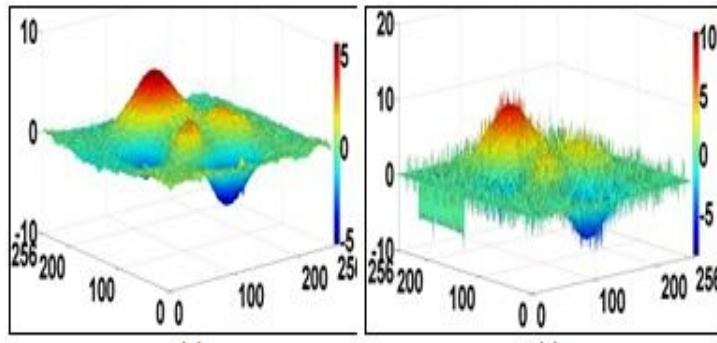

(a)

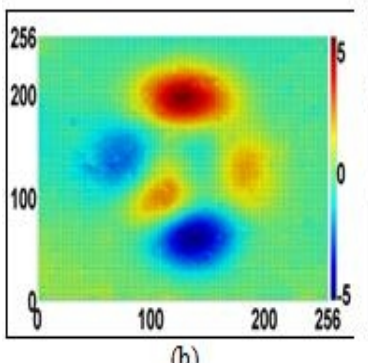

(b)

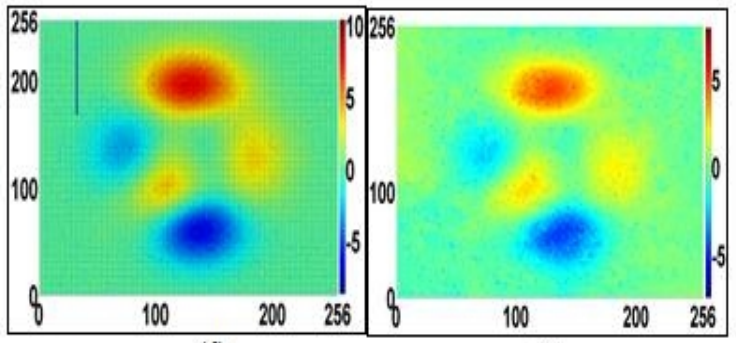

(d)

(f)

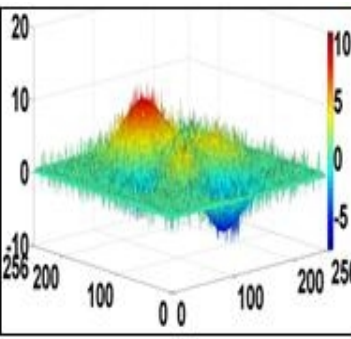

(e)

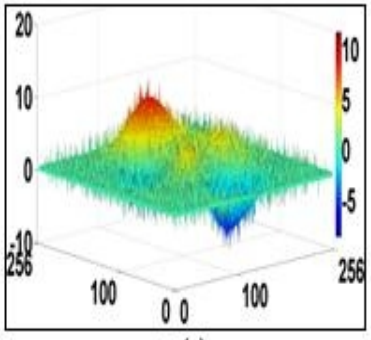

(g)

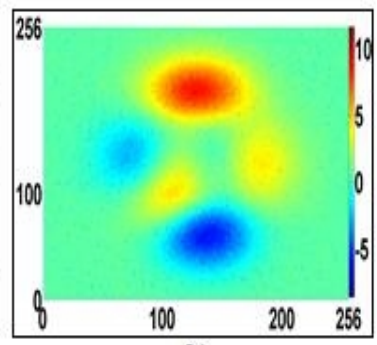

(h)

Fig.5 (a) result of the new algorithm,(b) top view of (a);(c) result of the NF,(d) top view of (c);(e) result of the LS,(f) top view of (e);(g) result of the $\mathrm{BC}$,(h) top view of (g)

From the 2D diagram of four kinds of unwrapping algorithms in Fig. 5, compared with the original phase $2 \mathrm{D}$ diagram, we can see that since the processing of the residual points is not processed, the noise is propagating in the global range, making the least squares method the most ambiguous. The integral path planning of the branch cutting method and the network flow method makes the influence of the residual point significantly reduced. The new method is effective in estimating the residual points. Although there is still a phenomenon of noise diffusion, the effect of noise is effectively consistent. From the results of the four methods can see 3D branch cut flow method and least square method, network results in the obvious noise points, the 3D image appeared obvious 'spikes' phenomenon. The new method effectively inhibits the effect of noise, making the 3D results present a good phase surface. From the result summary table, it can be seen that the cutting method consumes the longest time, but the error is not the smallest. The operation time of the network flow method is similar to the branch cutting method, but the error is the smallest. The running time of the least square method is shorter than the other methods, but the error is the most. The running time of the new 
method is longer than that of the least square method, and the root mean square error is much shorter than that of the branch cutting method and the network flow method. The root mean square error is much smaller than that of the branch cut method and the least square method.

Fig.6 (a) is the wrapped surface with SNR of $-0.51 \mathrm{~dB}$, and Fig.6 (b) is the top view of (a). The unwrapped results are shown in Fig.7, and the experiment results are summarized in Table 2.

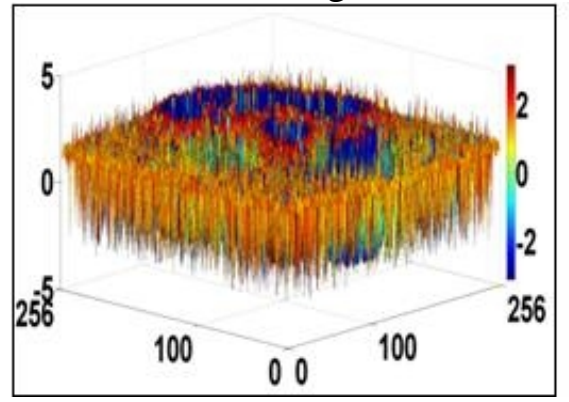

(a)

Fig.6 (a) wrapped phase with SNR of -0.51dB;

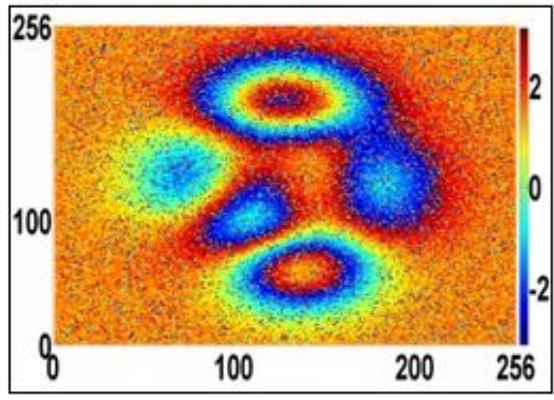

(b)

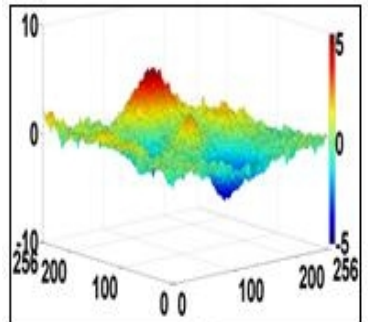

(a)

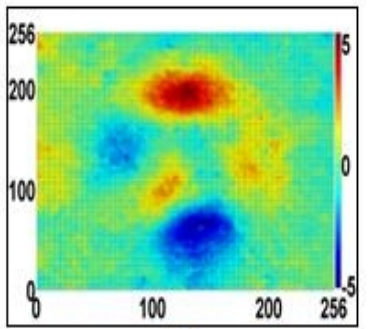

(b)

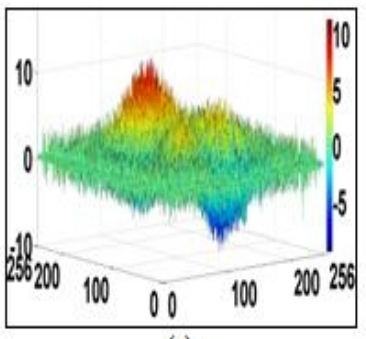

(c)

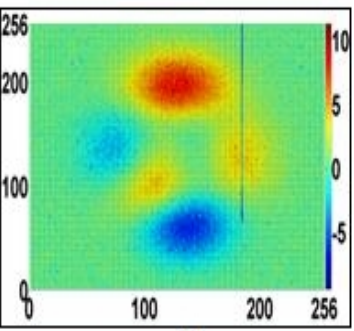

(d)

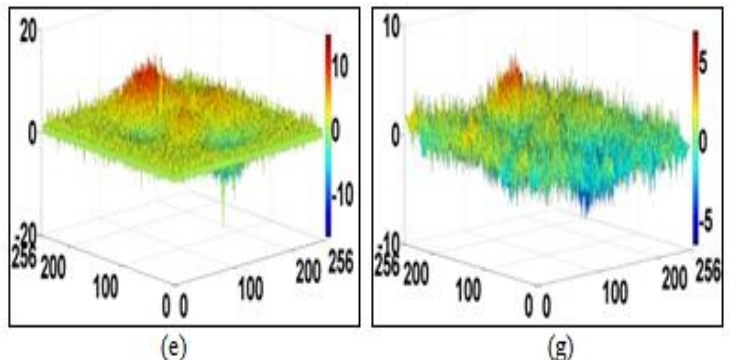

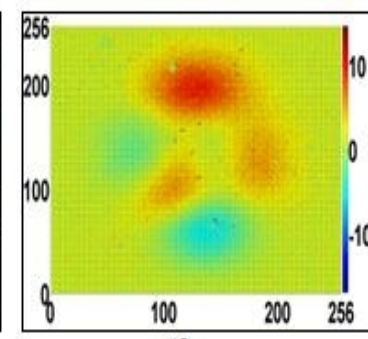

(f)

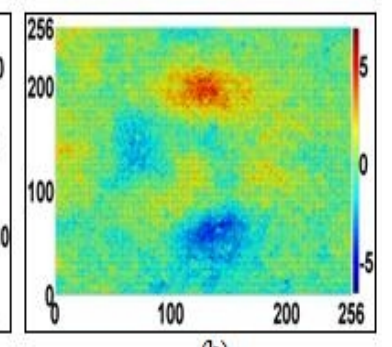

(h)

Fig. 7 (a) result of the new algorithm,(b) top view of (a),(c) result of the NF,(d) top view of (c),(e) result of the LS,(f) top view of (e),(g) result of the BC,(h) top view of (g)

From the results of four kinds of unwrapping algorithms in Figure 7, we can see that the four methods show the same characteristics for different SNR data. The results of branch cut method and least square method show that the 2D diagram is the most ambiguous, and the noise propagation in the global range can be clearly seen from figure 7(f) and 7(h). The unwrapping results of the new algorithm show a good suppression of the noise.

Table 2 simulation results with SNR of $-0.51 \mathrm{~dB}$

\begin{tabular}{|l|c|c|}
\hline Algorithm & Running Time(s) & RMSE \\
\hline branch cut & 7.2852 & 1.7222 \\
\hline least square & 0.2350 & 1.4374 \\
\hline network flow & 5.4288 & 0.9786 \\
\hline new algorithm & 1.9436 & 1.0466 \\
\hline
\end{tabular}

Unwrapped results of the measured data: The experimental data comes from the ASAR data provided by the official website of European Space Agency, capturing an earthquake in Aquila Italy in 
2009. Using the ASAR data of Orbit No.36756 (before earthquake) on March 11 and Orbit No. 37257(after earthquake) on April 15, the phase interference result of the featured area (shown in Fig.8 (a)) is obtained by image registration and interferogram formation which is processed by the software Next ESA SAR Toolbox without multilooking. Then the interferogram is tailored to a $512 * 512$ image shown in Fig.8 (b).The image will serve as the data source in the following experiment.

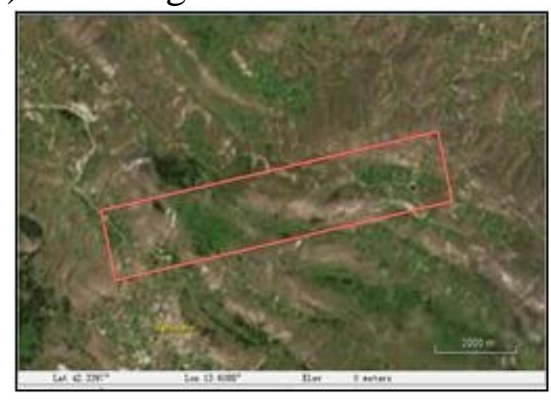

(a)

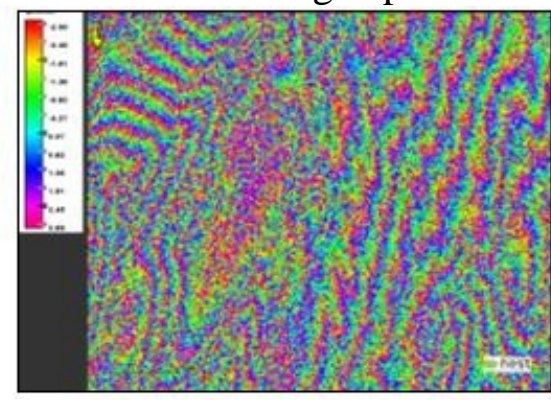

(b)

Fig. 8 (a) featured area in the world map

(b) the interferogram

Since the experimental data lacks the original continuous phase information, it is difficult to compare the phase root-mean-square error of the unwrapped results between different methods. Therefore, for the measured data, we use the rewrapping phase root-mean-square deviation $\sigma$ as the evaluation criteria in formula (13), which is the deviation between the rewrapping of the unwrapped phase and the original wrapped phase [10]. The deviation is considered as an important measurement of evaluating the unwrapping accuracy, estimating the ability of maintaining the phase values before and after implementing the algorithm. In formula (13), $\Phi_{i, j}^{w}$ represents the reverse wrapped phase. The unwrapped results of the four methods are shown in Fig.9, and the simulation results are summarized in Table 3.

$$
\sigma=\sqrt{\frac{\sum_{i=0}^{M} \sum_{j=0}^{-1}\left(\phi_{i, j}-\Phi_{i, j}^{w}\right)^{2}}{M \times N}}
$$

Table 3 Measured data results

\begin{tabular}{|l|c|c|}
\hline Algorithm & Running Time $(s)$ & $\sigma$ \\
\hline branch cut & 127.6868 & --- \\
\hline least square & 1.9764 & 2.5740 \\
\hline network flow & 48.9219 & 3.0344 \\
\hline new algorithm & 8.3976 & 2.4780 \\
\hline
\end{tabular}



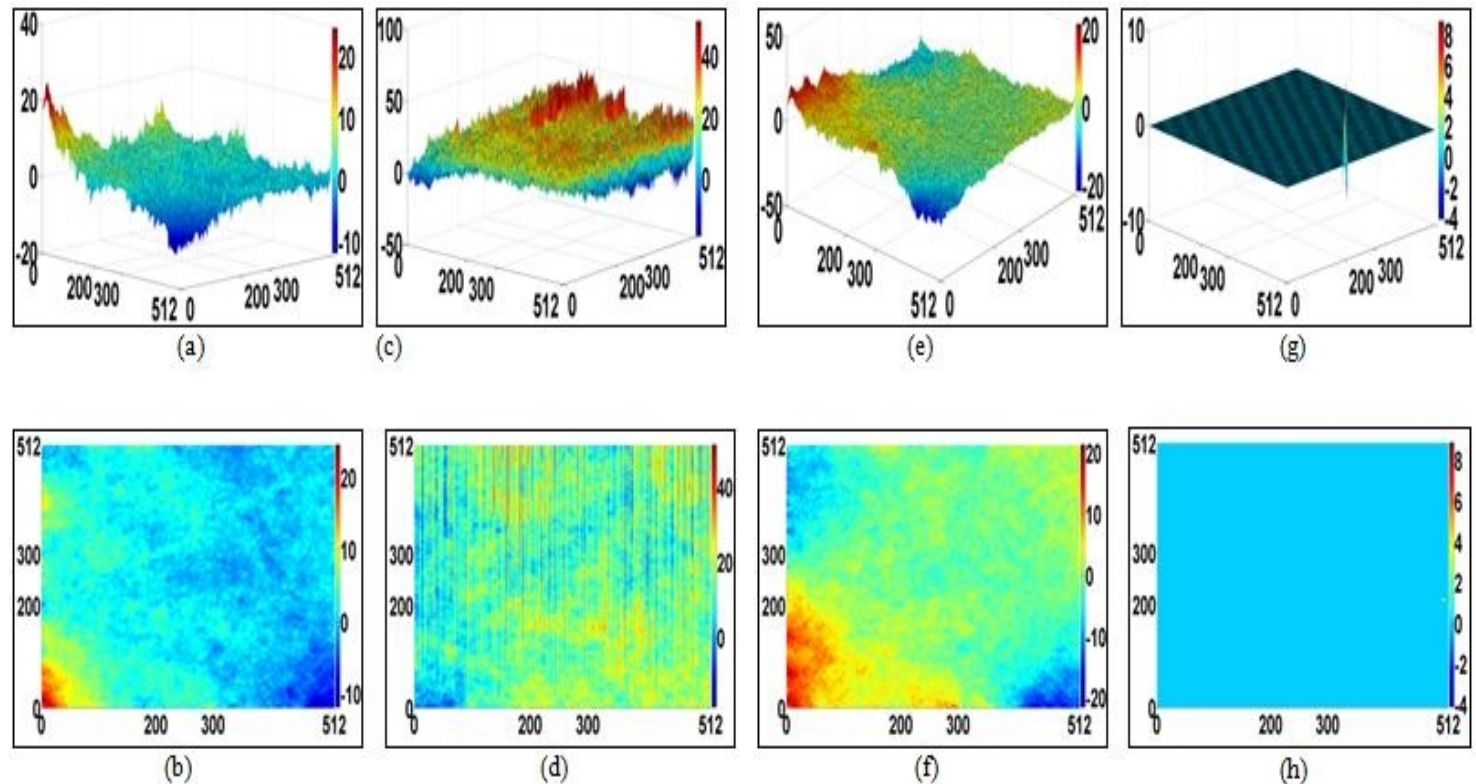

Fig.9 (a) result of the new algorithm,(b) top view of (a);(c) result of the NF,(d) top view of (c);(e) result of the LS,(f) top view of (e);(g) result of the BC,(h) top view of (g)

From the experimental results Figure 5, Figure 7 and Figure 9, we can see that: When there are many residual points, the 'island' is easy to appear in the unwrapping result of the branch cutting method, and its running time is the longest. Because there are too many residual points in measured data, the dendrite unwrapping result graph 9 (a) is obviously wrong. From the 2D diagram of three different data unwrapping results, it is obvious that the least square method takes the shortest time for unwrapping, but the precision of unwrapping results is low. The network flow algorithm effectively avoids the appearance of the 'island', and has a good reduction for the phase surface. However, there are a lot of spike burrs, which affect the accuracy of the results. The results of the new algorithm are the best for the reduction of the phase surface, and the peak spikes and glitches of the network flow results is improved effectively. From the summary of Table 1, table 2 and table 3, we can see that the least speed of the least square method is the fastest, but its error is the largest. The operation time of the branch cutting method is much longer than that of the least square method, and the accuracy of the unwrapping result is lower than the network flow, and there are phenomenons of unwrapping failure. The speed of the network flow algorithm is the same as the branch cut method. The running time of the new algorithm is much shorter than that of branch cutting and network flow. The accuracy of the algorithm is much higher than that of the least square method, and it also effectively inhibits the burr phenomenon in the entangled result.

\section{Conclusion}

In this paper, a new phase unwrapping algorithm is proposed to improve the noise influence in the unwrapping process. The method of this paper is based on the least square method, and the step of identifying the residual points is added to the unwrapping step. A local frequency estimation method is applied to fixed-point processing of the residual points caused by the noise, and a small time cost is used to reliably estimate residual error errors caused by noise. The new method effectively inhibits the error in the global range and improves the precision of the entangling. The new unwrapping algorithm is more accurate than the least square method and the branch cutting method, and can effectively restrain the spikes. The new method is characterized by low precision and high precision, which is beneficial to the implementation of the project. The phase unwrapping accuracy is greatly increased from both the simulated data experiment and the real data experiment. The reliability of the method is proved and it has some advantages compared with other methods. 


\section{Acknowledgements}

This work was financially supported by the National Nature Science Foundation of China (61561010), Natural Science Foundation of Guangxi Province of China (2017GXNSFAA198089), the Scientific research and technology development plan project of Guangxi Province of China (14122006-6),Scientific research project of Guangxi Education Department (KY2015LX096).

\section{References}

[1] Xing, Shuo, and Hongwei Guo.: 'Temporal phase unwrapping for fringe projection profilometry aided by recursion of Chebyshev polynomials', Applied Optics, Vol. 56(6), pp. 1591-1602, Feb,2017.

[2] Shevkunov, Igor.: 'A new phase unwrapping method', Journal of Physics: Conference Series. Vol. 737. IOP Publishing, 2016.

[3] Yu, Hanwen, and Yang Lan.: 'Robust Two-Dimensional Phase Unwrapping for Multibaseline SAR Interferograms: A Two-Stage Programming Approach',IEEE Trans. Geosci. Remote Sensing, vol.54, pp.5217-5225,2016.

[4] R. M. Goldstein, H. A. Zebker, and C. L. Werner: 'Satellite radar interferometry:

Two-dimensional-dimensional phase unwrapping', Radio Sci., vol. 23, pp. 713-720, 1988.

[5] Bioucas-Dias, Jos M., and Gonalo Valadao: 'Phase unwrapping via graph cuts', IEEE

Transactions on Image processing, vol.16, pp. 698-709, 2007.

[6] Fornaro, Gianfranco, and Eugenio Sansosti: 'A two-dimensional region growing least squares phase unwrapping algorithm for interferometric SAR processing', IEEE transactions on geoscience and remote sensing, vol.37, pp. 2215-2226, 1999.

[7] Zebker, Howard A., and Yanping Lu: 'Phase unwrapping algorithms for radar interferometry: residue-cut, least-squares, and synthesis algorithms', JOSA A ,vol.15, pp.586-598,1998.

[8] M. Costantini: 'A novel phase unwrapping method based on network programming', IEEE Trans. Geosci. Remote Sensing, vol. 36, pp.813-821, May 1998.

[9] Baselice, F., Budillon, A., Ferraioli, G., \& Pascazio, V. (2014). Multibaseline sar interferometry from complex data. Selected Topics in Applied Earth Observations \& Remote Sensing IEEE Journal of, 7(7), 2911-2918.

[10] Carballo G F, Fieguth P W. Probabilistic cost functions for network flow phase unwrapping[J]. IEEE

Transactions on Geoscience \& Remote Sensing, 2000, 38(5):2192-2201.

[11] Ghiglia, D. C., \& Romero, L. A. (1994). Robust two-dimensional weighted and unweighted phase unwrapping that uses fast transforms and iterative methods. J.opt.soc.am.a, 11(1), 107-117.

[12]Zhimin Liu, Experimental and comparative study of InSAR phase unwrapping algorithms[J]. Remote Sensing Information, 2012(2):71-76.

[13] Lei, Shuangyan, and Song Zhang: 'Digital sinusoidal fringe pattern generation: Defocusing binary patterns VS focusing sinusoidal patterns', Optics and Lasers in Engineering, vol. 48, pp. 561-569, 2010.

[14]Souza J C D, Oliveira M E, Santos P A M D. Branch-cut algorithm for optical phase unwrapping[J]. Optics Letters, 2015, 40(15):3456-3459.

[15] Xie X. Iterated unscented Kalman filter for phase unwrapping of interferometric fringes[J]. Optics Express, 2016, 24(17):18872. 